\title{
FLUIDOS SUPERCRÍTICOS NA INDÚSTRIA QUÍMICA: O PROCESSO DE DESASFALTAÇÃO A PROPANO
}

\author{
H. A. R. GOMES ${ }^{1}$, A. B. N. BRITO ${ }^{1}$ \\ ${ }^{1}$ Universidade Federal do Espírito Santo, Centro Universitário Norte do Espírito Santo, \\ Departamento de Engenharias e Tecnologia \\ E-mail para contato: ana.brito@ufes.br
}

RESUMO - Fluidos supercríticos são obtidos quando a temperatura e pressão a que são submetidos atingem um ponto crítico. Nessas condições, propriedades como solubilidade e densidade são facilmente controladas, possibilitando a aplicação desses fluidos em diversos processos da indústria química, tais como, extração, impregnação e formação de partículas, e obtendo excelentes rendimentos com baixos níveis de resíduos a serem tratados. Desta forma, este trabalho teve por objetivo simular o processo de desasfaltação com propano utilizando o software Aspen Plus V7.3. Obteve-se um rendimento global de $39 \%$ no processo convencional e de $77 \%$ no processo supercrítico, observando-se uma melhoria de $97 \%$ com o uso do fluido em seu estado supercrítico.

\section{INTRODUÇÃO}

A tecnologia supercrítica é aplicada, principalmente, nas indústrias alimentícia, farmacêutica e de cosméticos, gerando produtos de alta qualidade e baixa quantidade de resíduos a serem tratados. Porém, devido aos altos custos dos processos supercríticos, os produtos obtidos a partir dessa tecnologia têm alto preço de mercado, diminuindo a competitividade e limitando a aplicação desses processos na indústria.

Algumas vertentes mundiais para tecnologias limpas têm motivado a substituição dos processos convencionais, o que vem acentuando a substituição de processos convencionais por processos em condições supercríticas. Os produtos obtidos têm grande aceitação no mercado, e seu alto valor agregado compensa os investimentos realizados. (Kopcak, 2003).

Além disso, há uma tendência internacional de rejeição de produtos obtidos a partir de processos agressivos ao meio ambiente, e os processos supercríticos aparecem como uma alternativa sustentável com produtos de alto valor agregado (Azevedo, 2001).

\section{REVISÃO BIBLIOGRÁFICA}

\subsection{Definição}

Fluidos supercríticos são aqueles que estão localizados na região física acima do ponto crítico. 


\section{9 a 22 de outubro de 2014 \\ Florianópolis/SC}

Esse ponto corresponde ao valor mais elevado de pressão e temperatura no qual pode haver equilíbrio líquido-vapor. A partir desse ponto, a substância passa a ser constituída de uma única fase, dita supercrítica. Além disso, a substância não pode ser liquefeita sem que a temperatura e a pressão sejam reduzidas. A redução de apenas uma dessas duas propriedades não é capaz de alterar o estado físico da substância (Carrilho et. al., 2001; Mazoni, 2008).

\subsection{Propriedades}

No estado supercrítico, uma substância se encontra numa fase intermediária entre o líquido e o gás, reunindo as melhores características das duas fases.

As propriedades relacionadas à densidade e capacidade de solubilização de um fluido supercrítico aproximam-se das de um líquido, enquanto que as propriedades relacionadas ao transporte de matéria, como a difusividade e viscosidade, se aproximam das de um gás. Para esse trabalho, faz-se importante observar o comportamento de duas propriedades, a saber: densidade e solubilidade.

Densidade: A densidade de um fluido supercrítico se comporta de maneira diferente dos fluidos convencionais frente às variações de pressão e temperatura. A pressões moderadas, a densidade do fluido supercrítico se aproxima da de um gás, e, conforme a pressão é aumentada, a densidade desse fluido se aproxima da densidade de um líquido.

Conforme mostra a Figura 1a, nas imediações do ponto crítico (área hachurada), variações na pressão e na temperatura alteram significativamente a densidade do fluido sem que haja mudança de fase, fazendo com que esta região seja a melhor região para se trabalhar com esses fluidos. (Williams e Clifford, 2000).

Solubilidade: É a quantidade máxima de soluto que se dissolve em uma quantidade fixa de solvente a uma dada temperatura. Quando um soluto é dissolvido em um fluido supercrítico, ocorre a formação de clusters, que são agrupamentos de moléculas do solvente ao redor de moléculas do soluto. Esses clusters aumentam a densidade local do fluido devido à atração entre moléculas de soluto e de solvente. Em função disso, a solubilidade é maximizada em relação aos fluidos convencionais (Barnabé, 2008).

O aumento da pressão sobre um sistema soluto-solvente supercrítico aumenta a solubilidade, pois faz com que os clusters diminuam de tamanho, aumentando as interações entre as moléculas de solvente e as de soluto. $\mathrm{O}$ efeito da temperatura na solubilidade é conhecido como efeito retrógrado, pois, a pressões moderadas ou baixas, o aumento da temperatura diminui a solubilidade e, a altas pressões, o aumento da temperatura aumenta a solubilidade, conforme observado na Figura 1b. A solubilidade também pode ser aumentada adicionando-se à solução um cossolvente, que altera as interações moleculares da solução e acrescenta outras, elevando o poder de solubilização do fluido (Barnabé, 2008; Mazoni, 2008). 


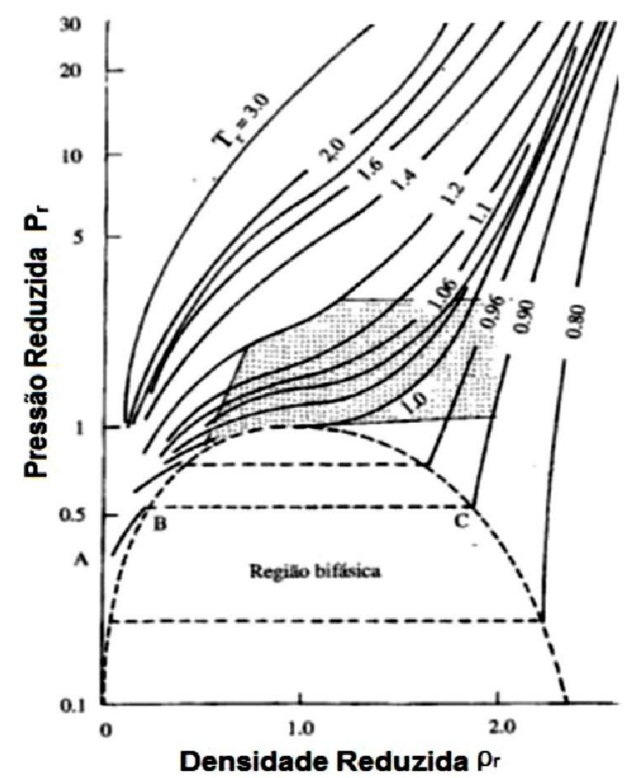

(a)

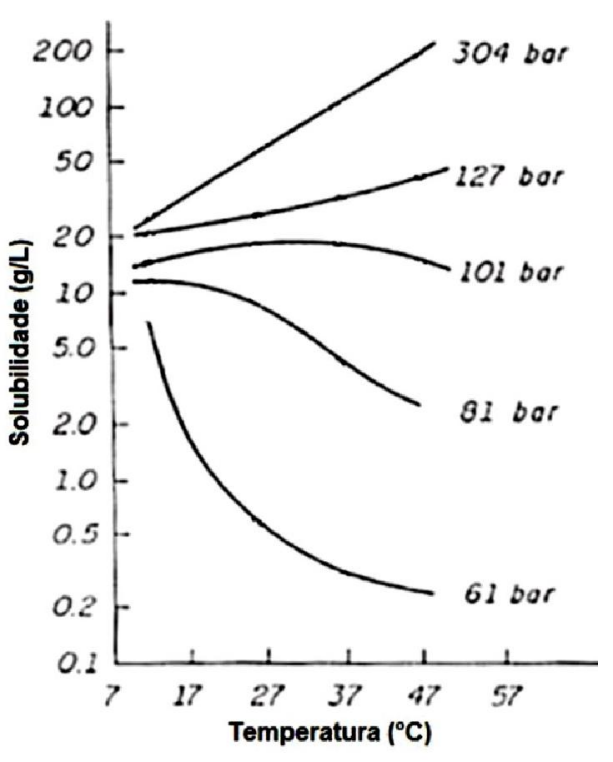

(b)

Figura 1 - (a) Curvas de pressão reduzida de uma substância em função da densidade e da temperatura reduzidas. (b) Curvas de solubilidade em função da temperatura.

\section{ESTUDO DE CASO: DESASFALTAÇÃO A PROPANO}

Os fluidos supercríticos possuem diversas aplicações na indústria, tais como, Impregnação em Meio Supercrítico, Formação de Partículas em Meio Supercrítico, Extração em Meio Supercrítico, Reação Química em Meio Supercrítico e Cromatografia Supercrítica. Os processos que utilizam esses fluidos são mais eficazes em termos de rendimento e qualidade do produto final, além de evitar o uso de solventes contaminantes e de prevenir resíduos indesejáveis ao final do processo.

Para melhor visualização das aplicações dos fluidos supercríticos, foi feito um estudo de caso usando a extração no processo de desasfaltação a propano. Esse processo pertence ao refinamento do petróleo. Nele, o propano solubiliza as cadeias parafínicas, ao passo que precipita os asfaltenos de uma corrente.

Foram simulados o processo em condições ambientes, o processo supercrítico e o processo supercrítico com adição de cossolvente. As três simulações foram feitas no software Aspen Plus ${ }^{\circledR}$ V7.3.

\subsection{Processo em Condições Ambientes}

O fluxograma para o processo em condições ambientes é mostrado na Figura 2, na qual se 
observa a corrente de entrada 1 , contendo uma mistura de compostos parafinicos e asfaltenos, e a corrente 2, contendo propano puro. Dentro do extrator (B1), o propano extrai as substâncias oleosas, que saem na corrente 3 . A corrente de rafinado, composta principalmente por asfaltenos deixa o processo pela corrente 4. A corrente 3 chega ao separador flash (B3), onde o soluto é recuperado (corrente 5) e o solvente (corrente 6) segue para o final do processo.

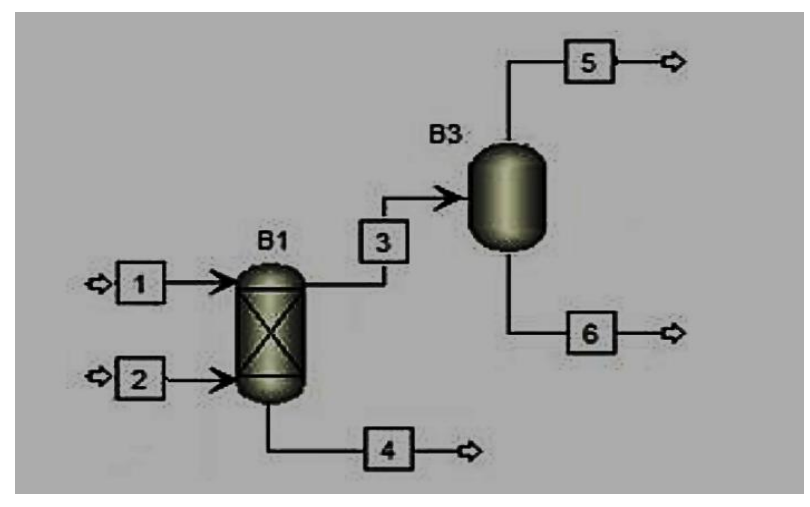

Figura 2 - Fluxograma para o processo de desasfaltação em condições ambientes.

Os dados de entrada são mostrados na Tabela 1 e os resultados obtidos para o processo nas condições ambientes são mostrados na Tabela 2.

Tabela 1 - Dados de entrada nas condições ambientes

\begin{tabular}{|c|c|c|}
\cline { 2 - 3 } \multicolumn{1}{c|}{} & \multicolumn{2}{c|}{ Corrente $(\mathrm{lbmol} / \mathrm{h})$} \\
\hline Substância & 1 & 2 \\
\hline Parafina & 100 & 0 \\
\hline Asfalteno & 300 & 0 \\
\hline Propano & 0 & 500 \\
\hline
\end{tabular}

Tabela 2 - Dados de saída nas condições ambientes

\begin{tabular}{|c|c|c|c|c|}
\cline { 2 - 5 } \multicolumn{1}{c|}{} & \multicolumn{4}{c|}{ Corrente $(\mathrm{lbmol} / \mathrm{h})$} \\
\hline Substância & 3 & 4 & 5 & 6 \\
\hline Parafina & 51,29 & 48,71 & 39,28 & 12,01 \\
\hline Asfalteno & 21,58 & 278,42 & 7,59 & 13,99 \\
\hline Propano & 492,34 & 7,66 & 17,01 & 475,33 \\
\hline
\end{tabular}

\subsection{Processo em Condições Supercríticas}

O fluxograma para esse processo é observado na Figura 3. A corrente 8 contém os asfaltenos e 
os parafínicos. A corrente 2 é a corrente de propano que foi levado às condições supercríticas por um trocador de calor (B1) e por uma bomba (B2). No extrator (B3), os asfaltenos são precipitados e saem na corrente 9. Os parafínicos são extraídos (corrente 3) e conduzidos a um trocador de calor (B4) e posteriormente a uma válvula (B5), os quais reduzem a pressão e a temperatura da mistura antes que esta entre no separador. Neste momento, o fluido não está mais nas condições supercríticas e o poder de solvência do propano é reduzido, fazendo com que o processo de separação tenha alta eficiência e proporcionando o reciclo da corrente 6 , que é novamente levada às condições supercríticas, originando a corrente 1 , que, por sua vez, gera a corrente 2 e o ciclo reinicia.

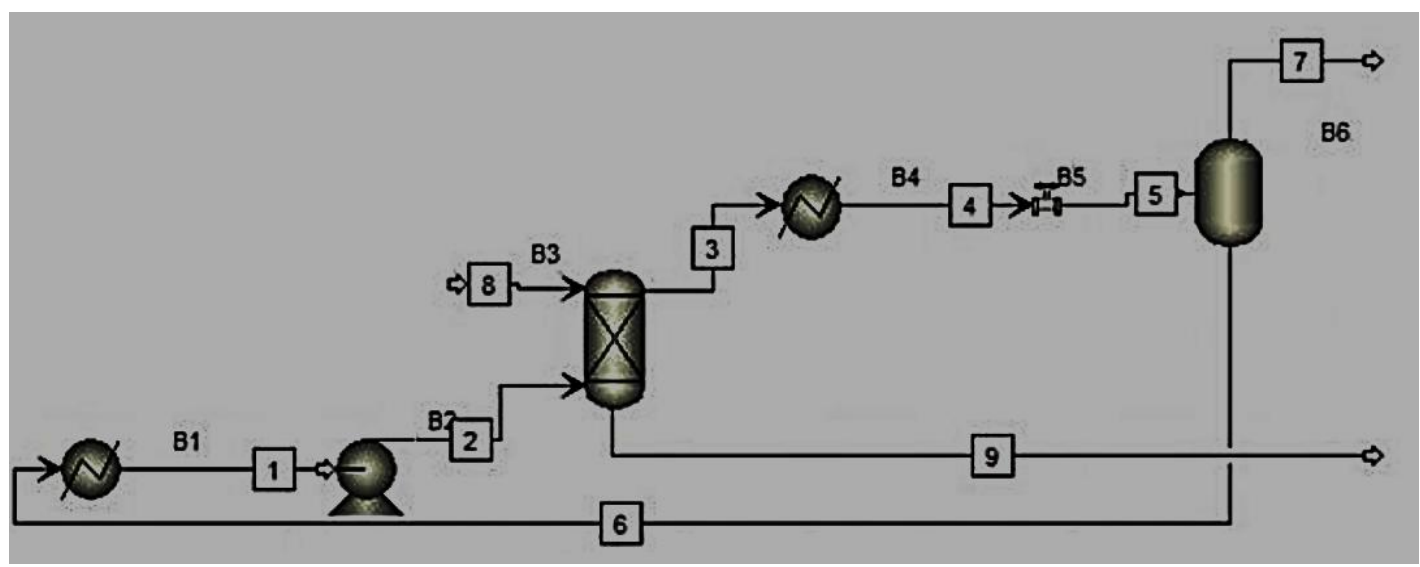

Figura 3 - Fluxograma para o processo em condições supercríticas.

Tabela 4.

As correntes de entrada são mostradas na Tabela 3 e as correntes de saída na

Tabela 3 - Dados de entrada nas condições supercríticas

\begin{tabular}{|c|c|c|}
\cline { 2 - 3 } \multicolumn{1}{c|}{} & \multicolumn{2}{c|}{ Corrente $(\mathrm{lbmol} / \mathrm{h})$} \\
\hline Substância & 8 & 2 \\
\hline Parafina & 100 & 1,82 \\
\hline Asfalteno & 300 & 2,24 \\
\hline Propano & 0 & 491,97 \\
\hline
\end{tabular}

Tabela 4 - Dados de saída nas condições supercríticas

\begin{tabular}{|c|c|c|c|c|}
\cline { 2 - 5 } \multicolumn{1}{c|}{} & \multicolumn{4}{c|}{ Corrente (lbmol/h) } \\
\hline Substância & 3 & 9 & 7 & 6 \\
\hline Parafina & 78,67 & 21,33 & 76,85 & 1,82 \\
\hline Asfalteno & 19,00 & 281,00 & 16,76 & 2,24 \\
\hline Propano & 493,23 & 6,77 & 1,26 & 491,97 \\
\hline
\end{tabular}




\subsection{Processo em Condições Supercrítica com Adição de Cossolvente}

O fluxograma para esse processo também é observado na Figura 4. A diferença entre o processo supercrítico e o processo supercrítico com adição de cossolvente é que as correntes 1, 2, 3, 4, 5 e 6 contêm, além do que já foi exposto nos itens anteriores, o butano que foi adicionado ao processo para aumentar a capacidade de solubilização do solvente supercrítico. Para esse processo, os dados de entrada são observados na Tabela 5 e os de saída na Tabela 6 .

Tabela 5 - Dados de entrada nas condições supercríticas com cossolvente

\begin{tabular}{|c|c|c|}
\cline { 2 - 3 } \multicolumn{1}{c|}{} & \multicolumn{2}{c|}{ Corrente $(\mathrm{lbmol} / \mathrm{h})$} \\
\hline Substância & 8 & 2 \\
\hline Parafina & 100 & 1,82 \\
\hline Asfalteno & 300 & 2,24 \\
\hline Propano & 0 & 491,97 \\
\hline Butano & 0 & 90,22 \\
\hline
\end{tabular}

Tabela 6 - Dados de saída nas condições supercríticas com cossolvente

\begin{tabular}{|c|c|c|c|c|}
\cline { 2 - 5 } \multicolumn{1}{c|}{} & \multicolumn{4}{c|}{ Corrente $(\mathrm{lbmol} / \mathrm{h})$} \\
\hline Substância & 3 & 9 & 7 & 6 \\
\hline Parafina & 78,67 & 21,33 & 76,85 & 1,82 \\
\hline Asfalteno & 19,00 & 281,00 & 16,76 & 2,24 \\
\hline Propano & 493,23 & 6,77 & 1,26 & 491,97 \\
\hline Butano & 92,75 & 7,25 & 2,53 & 90,22 \\
\hline
\end{tabular}

\section{RESULTADOS E DISCUSSÕES}

A Tabela 7 mostra o rendimento global do processo, o rendimento do extrator e o rendimento do separador.

O rendimento global foi obtido comparando-se, para o processo em condições ambientes, a corrente 5 com a corrente 1 , e para as condições supercríticas, a corrente 7 com a corrente 8 . O rendimento do extrator foi obtido comparando-se, para o processo em condições ambientes, a corrente 3 com a corrente 1, e para as condições supercríticas, a corrente 3 com a corrente 8 . Já o rendimento do separador foi obtido comparando-se, para o processo em condições ambientes, a corrente 5 com a corrente 3 , e para as condições supercríticas, a corrente 7 com a corrente 3 .

Tabela 7 - Rendimentos do processo nas condições de simulação

\begin{tabular}{|c|c|c|c|}
\cline { 2 - 4 } \multicolumn{1}{c|}{} & \multicolumn{3}{c|}{ Rendimento (\%) } \\
\hline Processo & Global & Extrator & Separador \\
\hline Convencional & 39 & 51 & 77 \\
\hline Supercrítico & 77 & 79 & 98 \\
\hline Cossolvente & 80 & 83 & 97 \\
\hline
\end{tabular}




\section{9 a 22 de outubro de 2014 \\ Florianópolis/SC}

A partir desses resultados, pode-se observar que o processo supercrítico é muito superior ao convencional, principalmente em termos de rendimento, que praticamente dobrou entre um e outro processo. Além dessa vantagem, vale ressaltar que a corrente contendo propano e parafínicos é facilmente separada devido ao controle de solubilidade que pode ser feito pelo simples ajuste das variáveis termodinâmicas. Essa separação é tão eficiente, que o propano é reciclado no processo.

\section{CONCLUSÃO}

Desse trabalho, conclui-se que os fluidos supercríticos têm uma ampla aplicabilidade e a utilização da tecnologia supercrítica melhora a qualidade do produto final e diminui a quantidade de resíduos contaminantes a serem tratados ou descartados. A análise de caso foi primordial na análise quantitativa da superioridade que um processo supercrítico tem sobre um processo convencional, mostrando o aumento de rendimento no processo, bem como a possibilidade de reaproveitamento do solvente que no processo convencional não pode ser reaproveitado. Apesar do alto custo de implantação, a tecnologia supercrítica pode diminuir gastos com tratamentos de efluentes e aumentar o valor agregado aos produtos, que são obtidos com alta qualidade e pureza.

\section{REFERENCIAS}

BARNABÉ, E. C. Extração de pilocarpina e epiisopiloturina de jaborandi utilizando processos envolvendo solventes supercríticos. 2008. Dissertação (Mestrado em Engenharia de Processos) - Programa de Pós Graduação em Engenharia Química, Universidade de Campinas, Campinas, 2008.

BUENO, C. Z.; MORAES, A. M.; SOUSA, H.C.; BRAGA, M. E. M. "Efeitos do processamento com $\mathrm{CO} 2$ supercrítico em membranas porosas de quitosana-alginato". In: Congresso Latino Americano De Órgãos Artificiais E Biomateriais, 2012, Natal.

CARRILHO, E.; TAVARES, M. C. H.; LANÇAS, F. M. "Fluidos supercríticos em química analítica. I. Cromatografia com fluido supercrítico: conceitos termodinâmicos". Quím. Nova, São Paulo, v. 24, n. 4, jul/ago 2001.

KOPCAK, U. Extração de Cafeína das Sementes da Planta de Guaraná (Paullinia cupana) com Dióxido de Carbono Supercrítico e co-solventes. Dissertação (Mestrado em Engenharia de Processos) - Programa de Pós Graduação em Engenharia Química, Universidade de Campinas, Campinas, 2003.

MAZONI, J. N. O. Inativação de fungos e extração de azadiractina e óleo de sementes de nim (Azadirachta indica A. Juss) utilizando fluidos supercríticos. 2008. Dissertação (Mestrado em Engenharia de Processos) - Programa de Pós Graduação em Engenharia Química, Universidade de Campinas, Campinas, 2008.

MELLO JUNIOR., P. A. Fronteiras da Engenharia Química I. Rio de Janeiro: E-papers, 2005.

WILLIAMS, J. R.; CLIFFORD, A. Supercritical Fluid Methods and Protocols. Totowa: Humana Press, 2000. 